\title{
Effets du porte-greffe sur la réaction du cyprès à la maladie du chancre cortical à Seiridium cardinale
}

\author{
C Andréoli *, J Ponchet, E Mari \\ Station de botanique et de pathologie végétale, Inra, BP 2078, F-06600 Antibes, France
}

(Reçu le 30 juillet 1995; accepté le 23 septembre 1996)

\begin{abstract}
Summary - Rootstock effects on the reaction of cypress to Seiridium cardinale bark canker disease. Reciprocal grafting was carried out on Cupressus sempervirens resistant or susceptible clones previously grown from self-rooting. When the scions were $10 \mathrm{~mm}$ in diameter, they were inoculated and then infection by Seiridium cardinale was observed over 2 years. The rootstock had no noticeable effect on the basic behaviour of scions. The susceptible clones died while resistant clones survived. However, the reactions of resistant clones to disease and scarring varied according to the rootstock clone. Resistance was correlated with tree growth, namely to the vigour conferred to scions by the rootstock. Resistance and growth, both induced by meristematic activity, show a good linear regression. It would be interesting to select vigorous rootstock clones, in order to increase resistance or tolerance as a consequence of an earlier compartmentalization and decrease the heterogeneity resulting from grafting on seedlings at random. Such a progress would require a good knowledge of self-rooting techniques.
\end{abstract}

grafting / compartmentalization / canker / Cupressus sempervirens = cypress

Résumé - Des boutures et des greffes réciproques ont été réalisées entre 14 clones de Cupressus sempervirens, résistants ou sensibles, préalablement élevés à partir de leur bouturage (tableau 1). Après inoculation des greffons ayant atteint $10 \mathrm{~mm}$ de diamètre on a suivi l'infection des troncs par Seiridium cardinale, durant deux ans. Le porte-greffe n'a pas d'effet notable sur le comportement fondamental des greffons (fig 1) : les sensibles meurent, les résistants survivent. Cependant, on constate des modulations de réaction à la maladie mises en évidence par les indices de compartimentage (tableaux II et III). La résistance des greffons est d'autant plus stimulée que les porte-greffes induisent la meilleure croissance (vigueur) des troncs (tableaux IV, V, VI ; fig 2). En particulier, la cicatrisation d'un clone est correlée avec la croissance en diamètre (fig 3 ). La vigueur conférée par le porte-greffe pourrait être sélectionnée de manière à améliorer la cicatrisation des clones résistants ou tolérants, grâce à un compartimentage plus précoce. En outre, le clonage des porte-greffes permettrait d'éviter l'hétérogénéité résultant des greffages sur plants issus de semis et d'améliorer l'état sanitaire des cyprès d'ornement ou de brise-vent. Un tel progrès suppose une maîtrise du bouturage.

greffage / compartimentage / chancre / Cupressus sempervirens = cyprès

* Correspondance et tirés à part 


\section{INTRODUCTION}

Les clones de cyprès (Cupressus sempervirens $\mathrm{L}$ notamment) sélectionnés pour leur résistance à Seiridium cardinale (WAG) Sutton et Gibson sont multipliés en pépinière par greffage sur des semis de la même espèce, âgés de 8 à 10 mois. Ces porte-greffes, issus d'allogamie, ont un port et une croissance variables qui, à priori, peuvent entraîner une certaine hétérogénéité morphologique dans une population de greffons clonés. Les interactions entre greffon et porte-greffe sont inconnues chez le cyprès, notamment vis-à-vis de $S$ cardinale. Chez la vigne, les modifications physiologiques observées, après greffage, se traduisent par plus ou moins de vigueur. Selon Rives (1971), ce concept est intuitif et résulte de la vigueur propre du scion et de celle "conférée" par le porte-greffe. Le porte-greffe module ainsi la vigueur, la productivité, la précocité, les réactions au milieu, etc, du greffon. II permet aussi de soustraire un système radiculaire à l'attaque d'agents pathogènes (Phytophthora sp) ou de ravageurs (Phylloxera) installés dans le sol, dans le cas où les boutures s'avèrent sensibles.

Concernant la réceptivité à une maladie des organes aériens du greffon, Delas (1979) a montré que le porte-greffe intervient de façon significative, à la fois sur la vigueur du cépage de vigne Merlot et sur la sensibilité de ses baies à Botrytis cinerea. Raj et al (1973) avec 19 porte-greffes ont conclu que quatre d'entre eux augmentaient la vigueur, le rendement et la tolérance au virus de la tristeza chez la lime (Citrus aurantifolia). Dosba et al (1984) ont montré que l'extériorisation des symptômes de mycoplasmes (MLO) sur Prunus sp dépend du porte-greffe. L'accroissement du diamètre du greffon est stimulée par un porte-greffe adéquat dans le cas du manguier (Hussain et al, 1989), du châtaignier (Makmet, 1979), du poirier (Selim et al, 1975). L'accroissement de vigueur est également visible après micro-greffage in vitro sur vigne (Martin et Collas, 1992).

Dans le cas du cyprès, la bibliographie ne contenant aucune information, notre travail a donc eu pour objectif l'étude des effets du portegreffe, sur la croissance des greffons et sur leurs réactions à la maladie. $S$ cardinale creuse dans l'écorce une lésion nécrotique (Panconesi, $1979)$; il traverse et envahit rapidement le périderme et le parenchyme, plus lentement le phloème. II détruit le cambium, ce qui laisse une tâche brune goudronneuse en surface du xylème ; les trachéides du bois ne sont pas attaquées. Chez les sujets sensibles, la nécrose progresse de façon continue, avec des accélérations saisonnières, jusqu'à ceinturer le tronc.

Après une invasion corticale passive de quelques mois (Ponchet et Andréoli, 1984), les sujets résistants réagissent lors de la reprise de croissance printanière. La résistance, du type "compartmentalization» (Mullick, 1977), se manifeste par une zone de réaction de couleur rouille, périphérique à la nécrose, dans laquelle un néophellogène érige un néopériderme barrière qui enferme les tissus malades dans un «compartiment" (Ponchet et Andréoli, 1989, 1990). Ce "compartiment" est progressivement éliminé au fur et à mesure que le cambium restaure la structure corticale nouvelle.

\section{MATÉRIEL ET MÉTHODES}

Étant donné la lenteur du développement de l'hôte et de son parasite, le dispositif expérimental a dû être étalé sur cinq ans, du bouturage des porte-greffes jusqu'à la dissection des plants survivants.

\section{Matériel végétal}

Les risques de pertes aléatoires, nombreux au cours d'une longue expérience ont conduit à préparer le bouturage de 200 ramilles herbacées de 14 clones, sept résistants $(R)$, sept sensibles $(S)$ de $C$ sempervirens dans du sable siliceux, sous brumisation, fin juin.

Ces clones provenaient des collections de l'Inra, soit de la station d'amélioration des arbres forestiers d'Avignon (clones 136R, 140R, 143S, 146S, 155R, $157 \mathrm{R}, 159 \mathrm{~S}, 160 \mathrm{R}$ ) soit de la station de botanique et de pathologie végétale d'Antibes (3S, 4R, 5S, 9R, 42S, $78 \mathrm{~S})$. En décembre, les greffages par approche, réciproques sur ces boutures porte-greffes ont utilisé comme scions (greffons) des ramilles des mêmes ortets, de 10 à $15 \mathrm{~cm}$ de long. Des boutures non greffées $(B)$ ont été conservées comme références. Aucun clone au comportement intermédiaire entre $R$ et $S$, qualifié de tolérant, n'a été retenu pour cette expérience.

\section{Inoculations}

Deux années après le greffage, les troncs des plants subsistants ayant atteint un diamètre d'environ $2 \mathrm{~cm}$ au dessus de la greffe, soit à $5 \mathrm{~cm}$ du sol, ont été inoculés en deux sites superposés sur une même génératrice du tronc, exposée au nord, pour éviter l'hétérogénéité des infections, induites par l'insolation. 
Les inoculations, l'une à $15 \mathrm{~cm}$, l'autre à $30 \mathrm{~cm}$ au dessus du point de greffe ont consisté à introduire dans des perforations de l'écorce des fragments de paille de sorgho infestés avec la souche de $S$ cardinale ATCC 38654 (Ponchet et Andréoli, 1984).

Ces deux niveaux étaient supposés servir de répétitions du facteur maladie. En outre, ils étaient de facto, d'âges différents : 4 ans pour le niveau bas, trois ans pour le plus élevé.

\section{Notations, paramètres mesurés}

- Hauteur $(\mathrm{H})$ des troncs et leur diamètre (D) au dessous de l'inoculation basse, soit entre 10 et $15 \mathrm{~cm}$ audessus de la greffe. Ils traduisent la plus ou moins grande vigueur des arbres.

- Longueur (LE) et largeur (WE) de l'empreinte qui matérialise, sur le rhitidome, la partie restaurée de l'écorce et donc la réactivité des clones $R$ (fig 1A).

Chez les clones $S$ l'accroissement de LE et WE est continu, les arbres se désséchant de manière échelonnée et permettant d'établir le pourcentage cumulé dans le temps de la mortalité des clones S (fig 2).

- Efficacité du compartimentage. La lésion corticale, de forme naviculaire, de dimensions superficielles LE et WE possède une épaisseur au plus égale à celle de l'écorce ; on ne peut la mesurer que sous microscope, sur coupes transversales. Lorsque la nécrose atteint le bois, elle laisse en surface un dépôt brun, goudron-

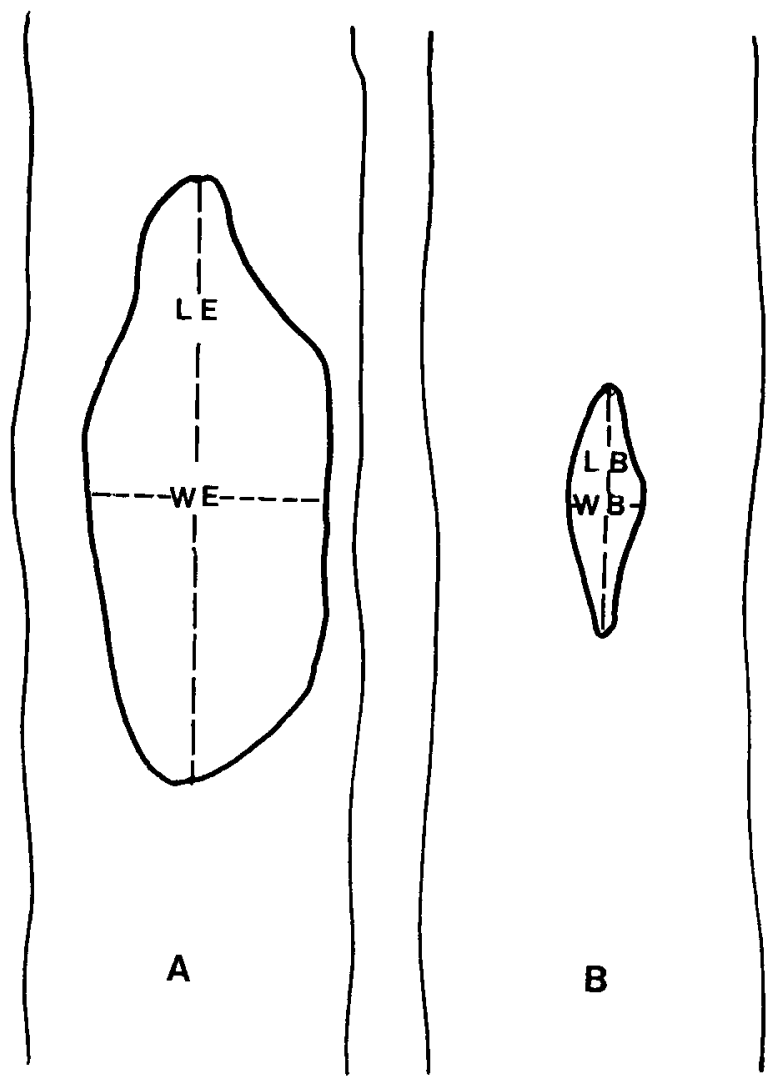

Fig 1. Empreinte de la nécrose corticale à $S$ cardinale (A) et de sa pénétration jusqu'au bois (B) et dimensions des paramètres LE, LB, WE, WB. neux dont on peut mesurer, après ablation de l'écorce nécrosée les dimensions : LB et WB (fig 1B). Ces dernières sont d'autant plus petites, par rapport à LE et WE que la réaction de compartimentage a été précoce et efficace ; elles peuvent être nulles. Les degrés de cette réaction ont été appréciés par deux indices de compartimentage :

$$
I C L=100 \frac{L E-L B}{L E}
$$

dans la longueur du tronc et

$$
I C W=100 \frac{W E-W B}{W E}
$$

pour la réaction en largueur. Ces indices sont d'autant plus élevés que la résistance est grande. Ils évaluent la capacité de résistance en minimisant la variabilité individuelle de LE et WE dont les valeurs absolues ne traduisent pas objectivement la réaction de l'hôte. ICL et ICW ne concernent pas les clones $S$ dont les lésions sont confluentes, l'écorce détruite et les arbres secs.

\section{Analyse statistique des résultats}

En s'inspirant du protocole proposé par Rives (1971, 1972) pour l'étude des multicombinaisons de porte-

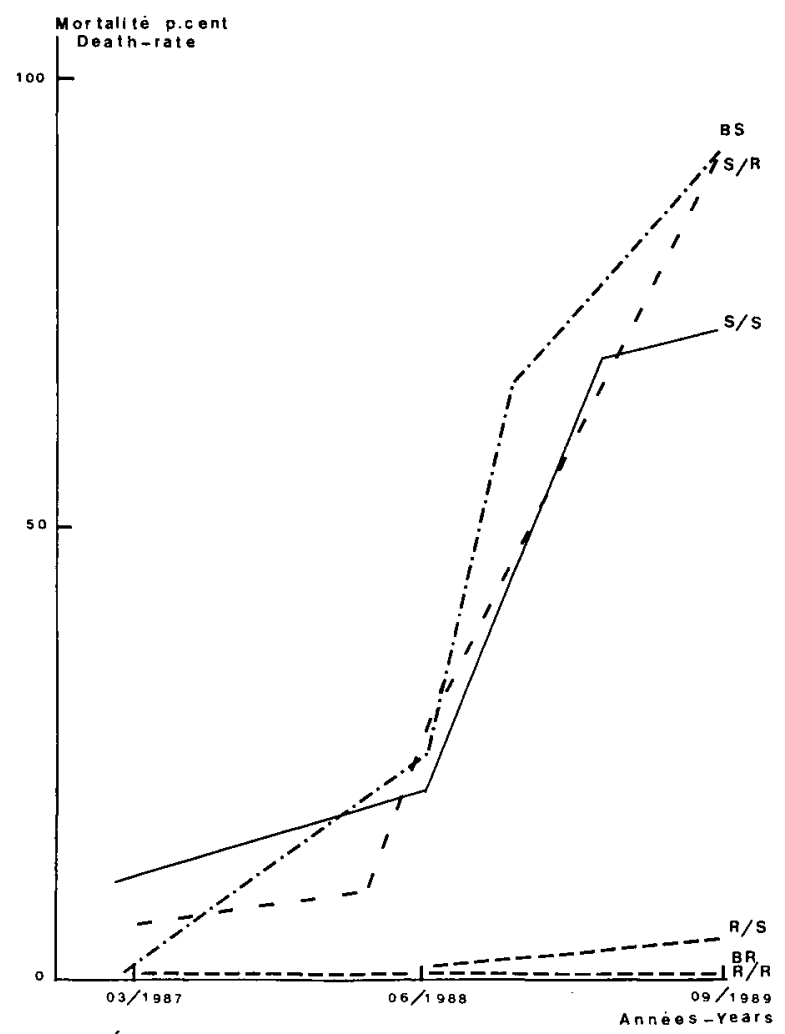

Fig 2. Évolution de la mortalité des scions sensibles à $S$ cardinale bouturés (BS), greffés sur sujets sensibles (S/S) ou résistants (S/R). Les scions résistants $B / R, R / S$ et $R / R$ se sont cicatrisés. 
greffes, on a analysé les variances, comparé les moyennes par le test de Tukey et calculé les régressions entre les variables. Le programme Systat Macintosh version 5,2, a été utilisé après que l'on se fut assuré de la normalité des distributions des quatre variables $I C L, I C W, D$ et $H$.

\section{RÉSULTATS}

Au moment des notations, les arbres mesuraient entre 130 et $230 \mathrm{~cm}$ (clone $140 \mathrm{R}$ ), entre 140 et $205 \mathrm{~cm}$ (clone 155R) et entre 120 et $170 \mathrm{~cm}$ (clone 160R). Les paramètres mesuraient de 40 à $100 \mathrm{~mm}$ (LE), 10 à $60 \mathrm{~mm}$ (LB), 15 à $45 \mathrm{~mm}$ (WE) et 01 à $12 \mathrm{~mm}$ (WB). Pour une lésion donnée cette variabilité importante est en partie absorbée dans le calcul de l'indice car il y a proportionnalité entre LE et LB ainsi qu'entre WE et WB.

\section{Comportement du matériel végétal et réactions à la maladie}

On trouvera dans le tableau I les performances des 14 clones utilisés et l'évolution de leurs effectifs.

Pour chacune des opérations successives, on constate de grandes différences entre clones pour l'aptitude au bouturage (B \%), comme porte-greffes (PG \%) ou comme scions ( $\mathrm{S} \%$ ).
Certains sont de mauvais porte-greffes mais des scions compatibles (157R, 146S) ou inversement (42 S, $78 \mathrm{~S}$ ). Tous les clones sensibles ont succombé à l'infection par $S$ cardinale sauf quatre rescapés du clone $3 \mathrm{~S}$. Les clones résistants ont achevé le compartimentage des lésions 18 mois après l'inoculation ; aucun d'entre eux n'est mort de la maladie qui ne s'est pas transmise au porte-greffe, même s'il était sensible.

La figure 2 montre l'évolution du pourcentage de mortalité des combinaisons avec des scions résistants : R/S, R/R et boutures $R$ ou des scions sensibles : $S / S, S / R$ et boutures $S$. Elle établit clairement que le comportement fondamental $R$ ou $S$ n'a pas été modifié.

Le dessèchement des scions $S$ a été sensiblement plus tardif sur les porte-greffes $42 S$ et $78 S$ que sur les autres. Les quatre arbres rescapés du scion $3 S$ (tableau I, fig 2 courbe $S / S$ ) étaient greffés sur $42 S$.

\section{Effets du porte-greffe sur le compartimentage}

Dans l'analyse de variance (tableau II) on a com. paré sur les trois porte-greffes $78 \mathrm{~S}, 42 \mathrm{~S}$ et $3 \mathrm{~S}$ les réactions des trois greffons 140R, 155R, 160R, seules combinaisons possédant, 5 ans après leur greffage et 18 mois après l'inoculation de quatre à huit arbres survivants, soit huit à 16 répétitions).

Tableau I. Aptitude des clones au bouturage (B\%) comme porte-greffes (PG\%) ou comme scions (S\%).

\begin{tabular}{llllll}
\hline Clone & $B(\%)$ & $P G(\%)$ & $S(\%)$ & INOC & \\
\hline & & & & \\
$4 \mathrm{R}$ & & & & & \\
$9 \mathrm{R}$ & 96 & 48 & 71 & 06 & 10 \\
$136 \mathrm{R}$ & 07 & 37 & 16 & 04 & 04 \\
$14 \mathrm{R}$ & 35 & 73 & 30 & 30 & 30 \\
$155 \mathrm{R}$ & 22 & 20 & 55 & 27 & 27 \\
$157 \mathrm{R}$ & 23 & - & 84 & 04 & 04 \\
$160 \mathrm{R}$ & 26 & 60 & - & 19 & 16 \\
$3 \mathrm{~S}$ & 22 & 51 & 36 & 05 & 04 \\
$5 \mathrm{~S}$ & 43 & 50 & 80 & 08 & 00 \\
$42 \mathrm{~S}$ & 92 & 71 & 53 & 07 & 00 \\
$78 \mathrm{~S}$ & 45 & 81 & 33 & 21 & 00 \\
$143 \mathrm{~S}$ & 68 & 45 & 63 & 12 & 00 \\
$146 \mathrm{~S}$ & 18 & - & 60 & 16 & 00 \\
$159 \mathrm{~S}$ & 03 & - & 47 & & 00 \\
\hline
\end{tabular}


D'après le tableau II, les niveaux d'inoculation se différencient ; le compartimentage étant plus actif au niveau bas qu'au niveau haut, à la fois pour ICL (moyenne 43,37 $\pm 2,13$ contre $37,08 \pm$ $2,15)$ et pour ICW $(86,55 \pm 1,15$ contre $82,69 \pm$ $1,18)$. Ces différences sont hautement significatives : $p=0,007$ pour ICL et $p=0,022$ pour ICW. II n'y a pas d'intéraction entre le niveau d'inoculation et les scions ou les porte-greffes. Les analyses ultérieures seront faites avec les indices du seul site d'inoculation basse (ICL ou ICW) puisqu'il n'est pas légitime de confondre les niveaux bas et haut. Dans le tableau II, les scions ne sont pas distincts, leurs réactions à $S$ cardinale étant voisines; en revanche il existe un effet significativement différent entre porte-greffes.

Le tableau III présente les moyennes ICL pour les combinaisons étudiées, l'analyse de variance donnant dans ce cas $p=0,149$ pour la source scion, $p<0,000$ pour la source porte-greffe et $p=0,468$ pour l'interaction scion porte-greffe.
L'effet porte-greffe provient uniquement du porte-greffe $42 S$ dont la moyenne d'après la matrice de Tukey est supérieure à celles du porte-greffe $78 \mathrm{~S}(p=0,003)$ du porte-greffe $3 S$ $(p=0,004)$ et des boutures $(p=0,001)$. Ces dernières ont la moyenne la plus basse par rapport aux porte-greffes 78S $(p=0,195)$ et $3 S$ $(p=0,403)$ qui eux sont égaux $(p=0,969)$.

Dans le détail, la comparaison par paires des moyennes du tableau III fait apparaître que la combinaison 140R/42S bénéficie de l'essentiel de l'effet porte-greffe en ayant un compartimentage supérieur à toutes les autres $(p<0,01)$ à l'exception de $160 \mathrm{R} / 42 \mathrm{~S}$ et de $155 \mathrm{R} / 42 \mathrm{~S}$ qui ne se distinguent pas entre elles, ni des autres combinaisons. L'effet du porte-greffe $42 S$ se confirme dans les détails comme le meilleur inducteur de cicatrisation des lésions de $S$ cardinale.

Si l'on considère, à titre complémentaire, les sites d'inoculation supérieurs, l'effet porte-greffe est également significatif : $p=0,002$ pour ICL et

Tableau II. Analyse de variance de l'effet porte-greffe sur la résistance à $S$ cardinale.

\begin{tabular}{|c|c|c|c|c|c|c|c|}
\hline \multirow[b]{2}{*}{ Sources } & \multirow[b]{2}{*}{$D L$} & \multicolumn{3}{|c|}{$I C L$} & \multicolumn{3}{|c|}{$I C W$} \\
\hline & & $C M$ & $F$ & Prob & $C M$ & $F$ & Prob \\
\hline Scion & 2 & 104,4 & 0,59 & 0,554 & 63,8 & 0,980 & 0,380 \\
\hline Porte-greffe & 3 & 1871,5 & 10,65 & 0,000 & 264,2 & 4,058 & 0,010 \\
\hline Niveau inoc & 1 & 1051,2 & 5,98 & 0,017 & 303,2 & 4,655 & 0,034 \\
\hline Scion $\times$ PG & 6 & 136,0 & 0,77 & 0,592 & 34,4 & 0,529 & 0,784 \\
\hline Scion $\times$ Niv inoc & 2 & 319,6 & 1,82 & 0,169 & 52,0 & 0,799 & 0,454 \\
\hline$P G \times N i v$ inoc & 3 & 238,6 & 1,36 & 0,262 & 59,9 & 0,920 & 0,435 \\
\hline Scion $\times$ PG $\times$ Niv inoc & 6 & 121,7 & 0,69 & 0,656 & 32,9 & 0,506 & 0,802 \\
\hline Erreur & 77 & 175,6 & & & 65,1 & & \\
\hline
\end{tabular}

Tableau III. Indices de compartimentage moyens (ICL) de l'inoculation inférieure pour les différentes combinaisons scion-porte-greffe.

\begin{tabular}{|c|c|c|c|c|c|c|c|c|}
\hline \multirow[t]{2}{*}{ Porte-greffe } & \multicolumn{2}{|c|}{ Scion 160} & \multicolumn{2}{|c|}{ Scion 140} & \multicolumn{2}{|c|}{ Scion 155} & \multicolumn{2}{|c|}{ Moyenne générale } \\
\hline & $I C L$ & $S E$ & $I C L$ & $S E$ & $I C L$ & $S E$ & $I C L$ & $S E$ \\
\hline $78 S$ & 34,5 & 5,9 & 42,0 & 5,1 & 47,5 & 5,5 & 41,3 & 3,2 \\
\hline $42 S$ & 49,0 & 7,2 & 74,0 & 7,3 & 61,6 & 6,5 & 61,5 & 4,04 \\
\hline $3 S$ & 27,0 & 8,4 & 43,4 & 6,5 & 44,7 & 7,2 & 38,4 & 4,35 \\
\hline Boutures & 37,5 & 10,2 & 31,5 & 10,3 & 26,5 & 10,3 & 31,8 & 5,9 \\
\hline Moyenne générale & 37,0 & 4,0 & 47,7 & 3,7 & 45,1 & 3,8 & & \\
\hline
\end{tabular}


$p=0,034$ pour ICW, le porte-greffe $42 S$ induisant toujours un compartimentage supérieur aux deux autres. II apparaît également que les boutures ont les moyennes les plus basses sans être significativement différentes de celles des portegreffes $78 \mathrm{~S}(p=0,346)$ et $3 \mathrm{~S}(p=0,469)$.

\section{Effet du porte-greffe sur la vigueur}

L'analyse des variances des paramètres $\mathrm{D}$ et $\mathrm{H}$ qui caractérisent la vigueur montre, pour chacun d'eux, un effet significatif des facteurs scion et porte-greffe $(p<0,000)$ mais pas d'interaction entre eux $(p=0,230)$. Le tableau IV donne les valeurs de $D$ des trois scions sur les trois portegreffes ; celles de $\mathrm{H}$ sont consignées dans le tableau $\mathrm{V}$.

Le scion 155R possède le meilleur développement tandis que $140 \mathrm{R}$ et $160 \mathrm{R}$ ont le même diamètre mais des hauteurs différentes.

Le porte-greffe $42 \mathrm{~S}$ confère aux trois scions une vigueur très significativement supérieure à celles induites par les porte-greffes $78 \mathrm{~S}$ et $3 \mathrm{~S}$ qui ne se distinguent pas entre eux. Le greffage est cependant bénéfique à la croissance des arbres, comparé au bouturage.

Les données du tableau $\mathrm{V}$ conduisent à des conclusions identiques à celles déduites du tableau IV. Croissance en hauteur et en diamètre sont proportionnelles avec des rapports $H / D$ moyens de 78,2 et 77,8 , pour les scions $160 \mathrm{R}$ et $155 R$, quel que soit le porte-greffe. Pour le scion 140R les rapports varient avec les porte-greffes de 75 à 100.

La vigueur conférée par $42 \mathrm{~S}$ aux scions s'extériorise donc surtout dans la croissance en diamètre du tronc.

\section{Relation entre le compartimentage et la vigueur}

Le greffage améliore à la fois le compartimentage et la vigueur des scions de trois clones de cyprès ; on peut donc se poser la question du

Tableau IV. Vigueur conférée au diamètre $(\mathrm{mm})$ des scions par les porte-greffes.

\begin{tabular}{|c|c|c|c|c|c|c|c|c|}
\hline \multirow[b]{3}{*}{$P G$} & \multicolumn{8}{|c|}{ Scion } \\
\hline & \multicolumn{2}{|c|}{$160 R$} & \multicolumn{2}{|c|}{$140 R$} & \multicolumn{2}{|c|}{$155 R$} & \multicolumn{2}{|c|}{ Moyennes } \\
\hline & $D$ & $S E$ & $D$ & $S E$ & $D$ & $S E$ & $D$ & $S E$ \\
\hline $78 S$ & 16,00 & 0,68 & 16,37 & 0,59 & 19,28 & 0,63 & 17,22 & 0,37 \\
\hline $42 S$ & 19,50 & 0,83 & 19,75 & 0,84 & 24,00 & 0,75 & 21,08 & 0,47 \\
\hline $3 S$ & 15,66 & 0,97 & 16,80 & 0,75 & 20,50 & 0,84 & 17,32 & 0,50 \\
\hline Boutures & 15,00 & 1,18 & 13,00 & 1,18 & 17,50 & 1,18 & 15,17 & 0,68 \\
\hline Moyennes & 16,29 & 0,47 & 16,48 & 0,43 & 20,32 & 0,44 & & \\
\hline
\end{tabular}

SE : erreur standard.

Tableau V. Vigueur conférée à la hauteur $(\mathrm{cm})$ des scions par les porte-greffes. SE erreur standard.

\begin{tabular}{|c|c|c|c|c|c|c|c|c|}
\hline \multirow[b]{3}{*}{$P G$} & \multicolumn{8}{|c|}{ Scion } \\
\hline & \multicolumn{2}{|c|}{$160 R$} & \multicolumn{2}{|c|}{$140 R$} & \multicolumn{2}{|c|}{$155 R$} & \multicolumn{2}{|c|}{ Moyennes } \\
\hline & $H$ & $S E$ & $H$ & $S E$ & $H$ & $S E$ & $H$ & $S E$ \\
\hline $78 S$ & 124,50 & 8,07 & 135,75 & 6,98 & 152,14 & 7,47 & 137,46 & 4,34 \\
\hline $42 S$ & 148,50 & 9,88 & 148,75 & 9,88 & 190,00 & 8,84 & 162,42 & 5,51 \\
\hline $3 S$ & 126,33 & 11,41 & 164,40 & 8,34 & 181,50 & 9,88 & 154,08 & 5,83 \\
\hline Boutures & 119,50 & 13,97 & 129,00 & 13,97 & 155,00 & 13,97 & 134,50 & 8,07 \\
\hline Moyennes & 127,21 & 5,52 & 144,47 & 5,12 & 169,66 & 5,16 & & \\
\hline
\end{tabular}


type de relation existant entre ces deux caractères en faisant l'hypothèse d'une corrélation, entre ICL ou ICW et D ou H.

Avec les données groupées des analyses précédentes on obtient les corrélations simples de la figure $3 A$.

Seule la composante ICL du compartimentage est significativement corrélée avec $D$ et $H$, alors que ICW ne l'est pas.

Dans le détail, il était intéressant d'étudier cette relation scion par scion en incorporant les données obtenues sur les porte-greffes autres que $78 \mathrm{~S}, 42 \mathrm{~S}$ et $3 \mathrm{~S}$ non communs aux trois scions (tableau I) à condition que leur effectif fût au moins égal à trois : fig $3 \mathrm{~B}$ pour le scion 140R, fig $3 \mathrm{C}$ pour le scion 155R.

Le tableau VI regroupe les coefficients de corrélation (CR) pour les diverses combinaisons et les probabilités $(P)$ correspondantes.

Il existe une bonne corrélation positive entre la cicatrisation des lésions et la vigueur de l'arbre ; la croissance en diamètre (D) est plus concernée dans cette relation que l'allongement $(H)$. II se confirme que, parmi les composantes du com-
Fig 3. Relations linéaires d'ensemble entre l'indice de compartimentage $(\mathrm{ICL})$ et les paramètres de la vigueur : diamètre (D) en $\mathrm{mm}$ et hauteur $(\mathrm{H})$ en $\mathrm{cm}$. A : Corrélation générale entre les trois greffons et les trois portegreffes. B : Corrélation entre le greffon $140 \mathrm{R}$ et tous les portegreffes utilisés. C : Corrélation entre le greffon $155 \mathrm{R}$ et tous les porte-greffes utilisés.
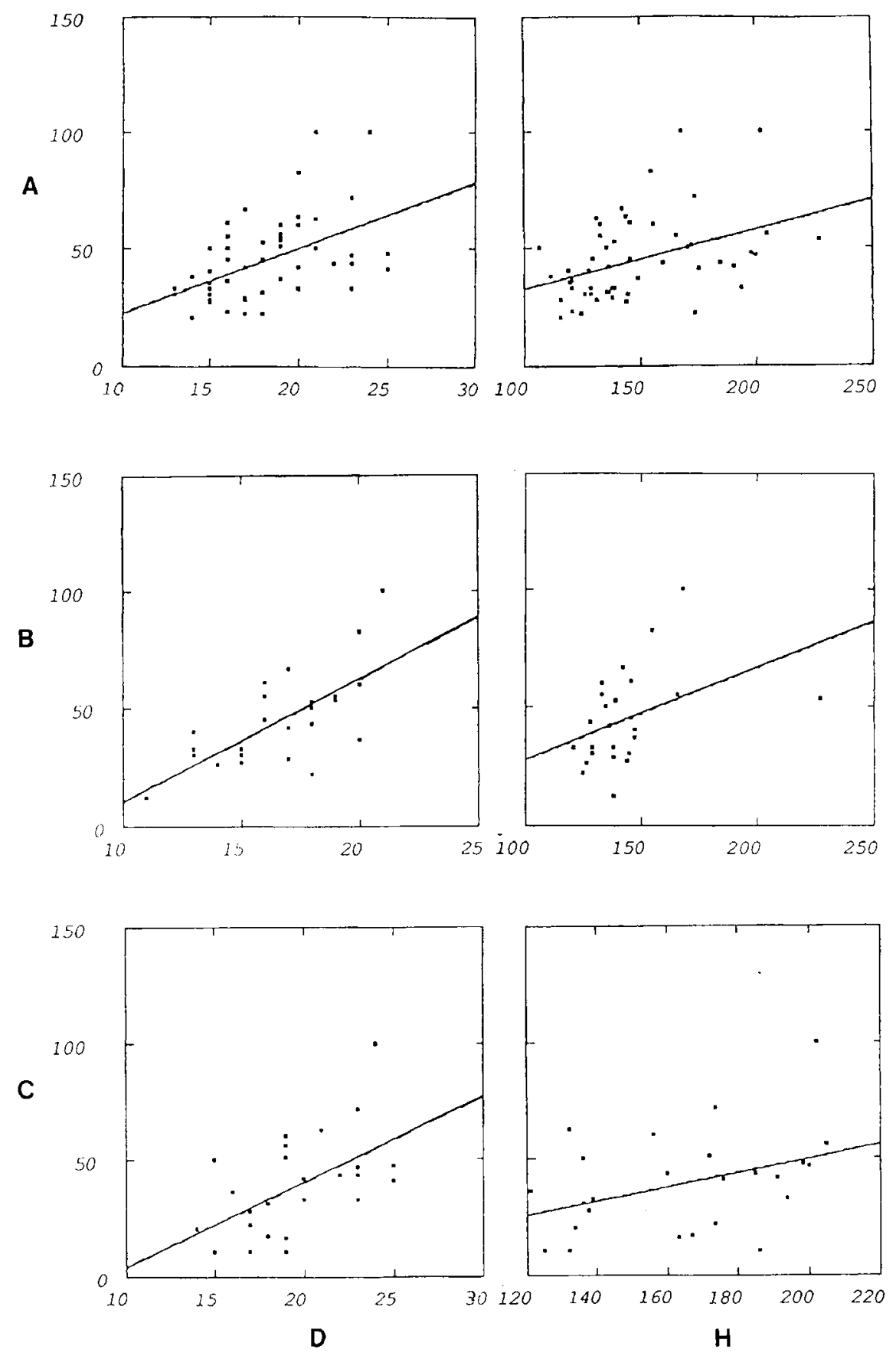
Tableau VI. Probabilités de correlation $(p)$ et coéfficients de regression (CR) entre compartimentage et vigueur.

\begin{tabular}{|c|c|c|c|c|c|c|c|c|}
\hline \multirow[t]{3}{*}{ Régression } & \multicolumn{8}{|c|}{ Scions } \\
\hline & \multicolumn{2}{|c|}{$160 R$} & \multicolumn{2}{|c|}{$140 R$} & \multicolumn{2}{|c|}{$155 R$} & \multicolumn{2}{|c|}{ Moyennes } \\
\hline & $C R$ & $P$ & $C R$ & $P$ & $C R$ & $P$ & $C R$ & $P$ \\
\hline ICL/D & 0,587 & 0,021 & 0,692 & $<0,000$ & 0,550 & 0,004 & 0,491 & $<0,000$ \\
\hline $\mathrm{ICL} / \mathrm{H}$ & 0,384 & 0,157 & 0,416 & 0,038 & 0,383 & 0,059 & 0,402 & 0,003 \\
\hline ICW/D & 0,244 & 0,381 & 0,479 & 0,015 & 0,544 & 0,005 & 0,215 & 0,127 \\
\hline ICW/H & 0,044 & 0,876 & 0,216 & 0,299 & 0,460 & 0,021 & 0,226 & 0,108 \\
\hline
\end{tabular}

partimentage l'indice ICL est le plus performant et le plus fiable.

\section{DISCUSSION ET CONCLUSIONS}

L'appréciation des réactions du cyprès à Seiridium cardinale, par une méthode quantitative, a permis de mettre en évidence les effets du porte-greffe sur la cicatrication de l'hôte tolérant ou résistant. Vu la plus grande importance de la longueur $(L)$ que de la largeur $(W)$ des nécroses induites par le pathogène dans l'écorce et dans le bois, l'indice qui lie les longueurs (ICL) bénéficie d'une plus grande précision que celui qui lie les largeurs (ICW).

La prise en considération des indices de compartimentage a permis aussi de démontrer clairement l'influence de l'âge des tissus corticaux sur le devenir des réactions de l'hôte, à deux niveaux d'inoculation ; influence controversée jusque là : Andréoli (1987), Ponchet et Andréoli (1990). Ainsi, le compartimentage des écorces de moins de deux ans est lent, parfois insuffisamment efficace, ce qui explique l'écimage fréquent des arbres par $S$ cardinale et justifie la nécessité, admise empiriquement, de ne pas inoculer des troncs d'un diamètre inférieur à $10 \mathrm{~mm}$ au risque de mal distinguer, la tolérance de la sensibilité.

Dans le cas d'un comportement "résistant" affirmé, les effets porte-greffe observés ne sont que des modulations d'un caractère génétique fondamental, polygénique, stable (Raddi et al, 1990). Le porte-greffe ne transforme pas cet état (fig 2). En revanche, le comportement «tolérant" mériterait une étude en liaison avec le greffage, ne serait-ce que pour expliquer sa relative instabilité et l'hétérogénéité des réponses de certains de ces clones à l'inoculation, après greffage sur semis tout venant.

La plupart des porte-greffes améliorent la cicatrisation des trois scions par rapport aux boutures mais quelques-uns (4R, 140R, 143S) ont tendance à l'atténuer. Seul, le porte-greffe $42 S$ améliore significativement le compartimentage des greffons en liaison avec la vigueur (diamètre). Cet effet additif résulte d'une vigueur conférée (Rives, 1972) ; les autres tendances de l'interaction porte-greffes/scions correspondent à des nuances de compatibilité. La vigueur conférée par le porte-greffe, comme le montre la corrélation positive ICL/D, se traduit surtout par l'accroissement du tronc en diamètre ; elle résulte d'une stimulation des méristèmes secondaires : phellogène et cambium, dans une plus faible mesure du méristème apical (corrélation ICL/H incertaine). Or, le processus de la réaction de résistance du cyprès à $S$ cardinale est, lui aussi, dépendant du produit des méristèmes secondaires : néopériderme barrière compartimentant la lésion à partir du phellogène, restauration de la structure par le cambium (Ponchet, Andréoli, 1981). Le mode d'action du porte-greffe sur la maladie est donc parfaitement logique et cohérent. Le processus anatomique de défense est modulé par la vigueur conférée au greffon par le porte-greffe.

La greffe étant le mode exclusif de multiplication végétative des clones de cyprès, il paraît tout à fait justifié de conduire une sélection de portegreffes à partir de critères simples : bonne croissance diamétrale, aptitude élevée et stable à la rhizogenèse (bouturage obligé), compatibilité avec une large gamme de scions. Un tel programme à long terme a été entrepris, parallèlement à une vérification de l'amplitude de la vigueur et de la résistance conférées aux scions, 
en comparaison avec leurs boutures ou leurs autogreffes.

La maîtrise du microbouturage du cyprès pourrait améliorer les résultats en permettant soit la multiplication des scions résistants, soit celle de porte-greffes performants, soit le microgreffage.

\section{RÉFÉRENCES}

Andréoli C (1987) Cypress age canker disease relationships. Proc 7th Congr Mediter Phytopathol Union, Granada, Espagne, sept 1987, 215-221

Delas J (1979) Comportement du merlot franc de pied et greffé en sol de Graves de la région de Bordeaux, influence de la fertilization azotée. Conn Vigne Vin 13, 115-124

Dosba F, Cassion F, Mazy K, Crossa-Raynaud P (1984) Le dépérissement de Molières : étiologie et comportement de différents prunus, $4^{\mathrm{e}}$ Colloque rech fruit, Bordeaux, 99-107

Hussain CM, Khan KM, Nasir MA (1989) Determination of suitable root-stock for some elite mango cultivars: J Agric Res (Lahore) 27, 289-298

Makmet BM, Katsalan VI (1979) Propagation of sweet chestnut by grafts for introduction (Ukrainian SSR). Bull Gl Bot Sada 114, 14-17

Martin C, Collas A (1992) De la culture in vitro à la production de greffés-soudés issus du greffage herbacé de la vigne. Progrès Agric Vit 109, 61-68

Mullick DB (1977) The non-specific nature of defence in bark and wood during wounding, insect and pathogen attack. Recent Adv Phytochem 2, 395441

Panconesi A (1979) II cancro del cipresso in Toscana : aspetti biologici. In : I/ cipresso: Malattie e difesa (V Grasso, P Raddi, eds), Seminario CEE Agrimed, Florence, Italie, 127-132

Ponchet J, Andréoli C (1984) Relations hôte-parasite dans le couple Cupressus-Coryneum cardinale. agronomie 4, 245-255

Ponchet J, Andréoli C (1989) Histopathologie du chancre cortical du cyprès à Seiridium cardinale. Eur J For Pathol 19, 212-221

Ponchet J, Andréoli C (1990) Compartmentalization and reaction in the host. In : Progress in EEC Research on Cypress Diseases (J Ponchet, ed), Report Eur 12 493, 96-111

Raddi P, Panconesi A, Xenopoulos S, Ferrandes P, Andreoli C (1990) Genetic improvement for resistance to canker disease. In : Progress in EEC. Research on Cypress Diseases (J Ponchet, ed), Report EUR 12 493, 127-136

Raj SA, Raja KTS, Durairaj P (1973) Role of root-stock on acid lime (Citrus aurantifolia Swingle decline), Annamalai Univ Agric Res Ann 4/5, 203-206

Rives M (1971) Statistical analysis of rootstock experiments as providing a definition of the terms vigour and affinity in grapes. Vitis 9, 280-290

Rives M (1972) Essais de porte-greffes multicombinaisons. Conn Vigne Vin 223-235

Selim HA, El-Azzouni MM, Fouad MM, El-Shurafa SY (1975) Stock-scion relationship in some compatible and incompatible unions of nursery pear plants. Egyptian J Hortic 2, 105-116 